\title{
Visualization of lenticulostriate arteries during insular low-grade glioma surgeries by navigated 3D ultrasound power Doppler: technical note
}

\author{
Andrej Šteňo, MD, PhD, ${ }^{1}$ Michaela Jezberová, MD, ${ }^{2}$ Vladimír Hollý, MD, ${ }^{3}$ \\ Gabriela Timárová, MD, PhD, ${ }^{4}$ and Juraj Šteňo, MD, PhD ${ }^{1}$ \\ 1Department of Neurosurgery and ${ }^{4} I I$. Department of Neurology, Comenius University, University Hospital Bratislava; ${ }^{2} \mathrm{Dr}$. Magnet \\ Kramáre, Dr. Magnet s.r.o.; and ${ }^{3}$ Department of Anesthesiology, Slovak Medical University, University Hospital Bratislava, \\ Slovakia
}

OBJECTIVE Resection of insular gliomas is challenging. In cases of intraoperative injury to the lenticulostriate arteries (LSAs), the usual result is a dense hemiplegia. LSAs are usually localized just behind the medial tumor border but they can also be encased by the tumor. Thus, exact localization of these perforators is important. However, intraoperative localization of LSAs using conventional neuronavigation can be difficult due to brain shift. In this paper, the authors present a novel method of intraoperative LSA visualization by navigated 3D ultrasound (3DUS) power Doppler. This technique enables almost real-time imaging of LSAs and evaluation of their shift during insular tumor resections.

METHODS Six patients harboring insular Grade II gliomas were consecutively operated on at the Department of Neurosurgery in Bratislava using visualization of LSAs by navigated 3DUS power Doppler. In all cases, the 3DUS data were repeatedly updated to compensate for the brain shift and display the actual position of LSAs and residual tumor.

RESULTS Successful visualization of LSAs was achieved in all cases. During all surgeries, the distance between the bottom of the resection cavity and LSAs could be accurately evaluated; in all tumors the resection approached the LSAs and only a minimal amount of tissue covering these perforators was intentionally left in place to avoid injury to them.

CONCLUSIONS Visualization of LSAs by navigated 3DUS power Doppler is a useful tool that may help to prevent injury of LSAs during removal of insular low-grade gliomas. However, reliability of this method has to be carefully evaluated in further studies.

http://thejns.org/doi/abs/10.3171/2015.10.JNS151907

KEY WORDS lenticulostriate arteries; glioma; insula; ultrasound; power Doppler; oncology

$\mathrm{O}$ NE of the most serious complications during resections of insular gliomas is injury to the lenticulostriate arteries (LSAs), which supply descending motor pathways. A dense hemiplegia is the usual result of LSA disruption. ${ }^{3}$ LSAs are usually localized just behind the medial tumor border, but they can also be encased by the tumor. ${ }^{9}$ These perforators represent the most medial limit of feasible tumor resection. Their intraoperative localization is therefore crucial, but it is challenging.,212 While LSAs may be preoperatively depicted by different imaging modalities, $, 9,14$ their intraoperative localization using conventional neuronavigation based on preoperative imaging can be difficult due to brain shift.

The aim of this paper is to present a novel method of intraoperative LSA localization based on navigated 3D ultrasound (3DUS) power Doppler imaging. In contrast to previous works that reported negative experiences with intraoperative LSA visualization using ultrasound Doppler techniques, ${ }^{2,5}$ we have observed that LSAs may be depicted by 3DUS power Doppler. This technique enables almost real-time intraoperative visualization of these important perforators and evaluation of their shift during the resection (Fig. 1), which may help prevent injury to them (Fig. 2).

Our first experience with visualization of LSAs using 3DUS power Doppler was during a second-look insular glioma awake surgery (after a partial resection performed elsewhere), terminated prematurely due to inadequate patient cooperation. Following this finding, navigated 3DUS

ABBREVIATIONS 3DUS = navigated 3D ultrasound; FLAIR = fluid-attenuated inversion recovery; LGG = low-grade glioma; LSA = lenticulostriate artery; MCA = middle cerebral artery; TOF = time of flight.

SUBMITTED August 14, 2015. ACCEPTED October 19, 2015.

INCLUDE WHEN CITING Published online February 5, 2016; DOI: 10.3171/2015.10.JNS151907. 

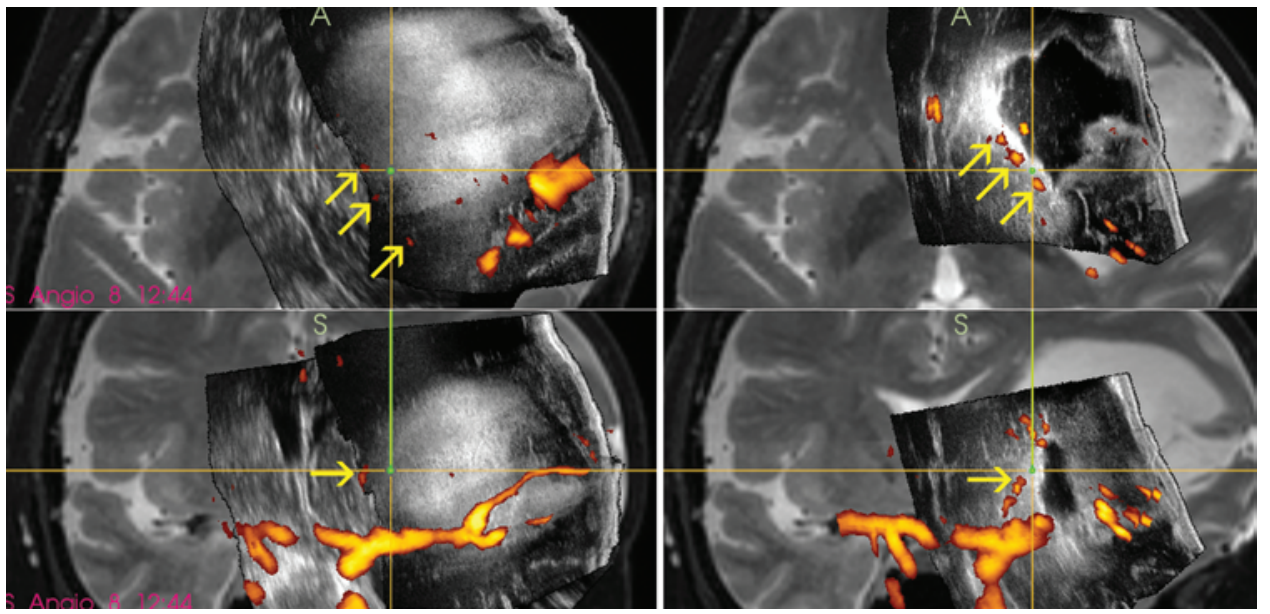

FIG. 1. Case 6. 3DUS power Doppler visualization of the LSAs (arrows) during insular tumor resection. Left: 3DUS image fused with MRI navigation sequence before resection. Right: 3DUS image acquired shortly before the end of resection. Note the significant shift of the LSAs: the center of the intersection of the yellow lines on both the left and right images is at an identical point of the cranial cavity; visualization of the shift prevented inadvertent exposure of the LSAs. Orange areas are vessels visualized by 3DUS power Doppler imaging.

power Doppler was regularly used during all insular glioma resections. In this paper we report our initial experience after first 6 consecutive surgeries.

\section{Methods}

Patient Population

Six patients who consecutively underwent operations for insular Grade II gliomas (low-grade glioma [LGG]) were included. The clinical features of these patients are detailed in Table 1.

All patients were preoperatively investigated by MRI using T1- and T2-weighted sequences, fluid-attenuated inversion recovery (FLAIR) sequences, contrast-enhanced T1-weighted sequences, perfusion-weighted imaging, and MR spectroscopy. Eloquent cortical and subcortical struc-
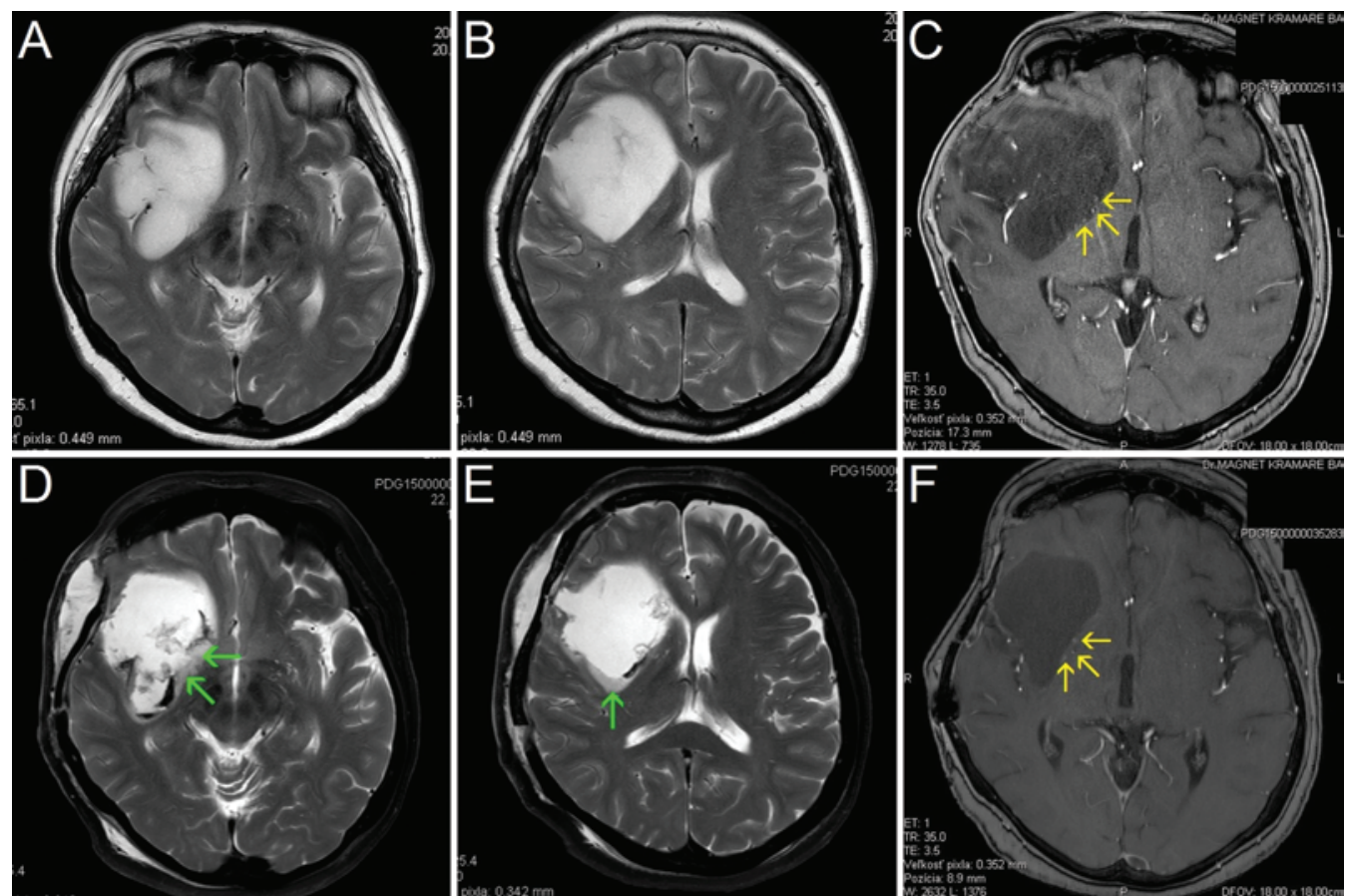

FIG. 2. Case 6. Pre- and postoperative axial MR images. A and B: Preoperative T2-weighted sequence showing a hyperintense insular tumor. C: Contrast-enhanced 3D TOF sequence showing the position of the LSAs (arrows); MRI was performed between the first and second stage of the surgery. D: Postoperative T2-weighted sequence showing subtotal resection. A minimal rim of tumor tissue was intentionally left in place (arrows) to avoid injuring the LSAs. E: Postoperative T2-weighted sequence showing residual tumor (arrow) close to the posterior arm of the internal capsule (mild reversible paresis of the left extremities occurred during resection in this area). F: Postoperative contrast-enhanced 3D TOF sequence showing the position of the LSAs (arrows) just under the resection cavity. 
tures were depicted by functional MRI and tractography based on diffusion tensor imaging. In the last 2 cases, a contrast-enhanced 3D time-of-flight (TOF) sequence was additionally used to display LSAs. Three-dimensional T2-weighted or 3D FLAIR MRI sequences were used for navigation.

\section{Operating Procedures}

All resections were performed under awake conditions. Direct electrical stimulation was used to identify eloquent (motor, speech, and language) cortex and white matter tracts.

A system that integrates standard neuronavigation and 3DUS and provides automatic fusion of both modalities (SonoWand Invite, SONOWAND AS) was used for navigation, for intraoperative evaluation of brain shift and extent of resection, as well as for intraoperative LSA visualization. After fixation of a patient's head in a Mayfield headrest, standard patient registration based on skin fiducials was performed. The initial ultrasound scan was performed on the dural surface. A flat-phased array probe (frequency range 3-8 MHz), large version of a flat linear array probe (frequency range $6-12 \mathrm{MHz}$ ), as well as the mini-craniotomy probe (frequency range $5-10 \mathrm{MHz}$ ) were used during the surgery. All probes were equipped with a reflective marker reference frame. The 3DUS data were acquired by freehand probe movement, tracking the position and orientation of the ultrasound probe by means of an integrated navigation camera system. LSAs were visualized using the power Doppler mode. To get the final intraoperative 3DUS image, an automatic fusion of power Doppler data acquired by phased array probe and anatomical data acquired by linear probe was performed. The mini-craniotomy probe was used mostly for minimization of acoustic enhancement artifacts, as described previously by our group..$^{17}$

During the surgeries, the ultrasound data were repeatedly updated to compensate for the brain shift and display the actual position of LSAs and tumor residua. The data update, including automatic image fusion, usually took approximately 2 minutes to complete.

Three surgeries (Cases 1-3) were planned as a singlestage procedure. The asleep-awake-asleep anesthetic technique $^{4}$ was used; general anesthesia was induced by using intravenously administered remifentanil and propofol, and a laryngeal mask was used to secure airways. The patients were woken up after the craniotomy. Despite planning as a single-stage procedure, resection in Case 1 was interrupted due to the technical difficulties with intraoperative ultrasound, and the patient in Case 3 reported a strong nausea shortly after the beginning of tumor removal, and therefore it was decided to terminate the surgery. In both preliminarily terminated cases, a successful awake resection was performed a few days later.

Three surgeries (Cases 4-6) were planned and performed as 2-stage procedures, according to the concept presented by Kumabe et al. ${ }^{6}$ Performing a craniotomy, drilling, and using rongeurs on the sphenoid wing, wide opening of the sylvian fissure, and resection of the anterior part of the temporal lobe (in case it was infiltrated) were performed under general anesthesia. The second surgery was performed a few days later, in which the entire procedure was performed under conscious sedation using dexmedetomidine and remifentanil. We prefer this approach for larger, especially dominant insular tumors (in which the resection duration is long, and typically takes several hours) to minimize the risk of suboptimal monitoring of neurological functions due to patient fatigue. In our experience, the resulting shortening of the duration of the procedure (due to insular portion removal starting shortly after the second surgery beginning) appears to allow the patients to fully cooperate until the end of tumor resection.

\section{Results}

In all cases, 3DUS power Doppler distinctly displayed LSAs (Fig. 3); the distance between the bottom of the resection cavity and LSAs could be accurately evaluated. In all tumors the resection stopped close to the LSAs and only a minimal amount of tissue covering these perforators was intentionally left in place, to avoid vascular injury. Five subtotal resections (residual tumor $<10 \mathrm{~cm}^{3}$ ) and 1 partial resection (Fig. 4) were achieved. The extent of resections and functional results are summarized in Table 1.

\section{Illustrative Case}

Case 5

This 23-year-old woman suffered 1-2 epileptic seizures

TABLE 1. Clinical details in 6 patients with insular gliomas

\begin{tabular}{|c|c|c|c|c|c|}
\hline $\begin{array}{l}\text { Case } \\
\text { No. }\end{array}$ & $\begin{array}{l}\text { Age (yrs), } \\
\text { Sex }\end{array}$ & Handedness/Clinical Presentation & Localization/Histology & $\begin{array}{c}\text { Preop tV }\left(\mathrm{cm}^{3}\right) / \text { Postop tV } \\
\left(\mathrm{cm}^{3}\right) / \mathrm{EOR}(\%)\end{array}$ & Postop Course \\
\hline 1 & $43, \mathrm{M}$ & Rt/seizures & Rt insula, FL, \& TL/All & $30.4 / 6.3 / 79.3$ & No new deficit \\
\hline 2 & $35, \mathrm{M}$ & Rt/seizures & Rt insula \& FL/OII & $71.6 / 1.2 / 98.3$ & No new deficit \\
\hline 3 & $19, \mathrm{~F}$ & Rt/headache & Lt insula \& FL/All & 13.8/1.6/88.4 & No new deficit \\
\hline 4 & $31, \mathrm{M}$ & $\begin{array}{l}\text { Rt/pharmacologically uncontrolled } \\
\text { seizures }\end{array}$ & Lt insula, FL, \& TL/All & $93.6 / 39.7 / 57.6$ & $\begin{array}{l}\text { No new deficit, seizure frequency } \\
\text { decreased }\end{array}$ \\
\hline 5 & $23, F$ & $\begin{array}{l}\text { Mixed/pharmacologically uncontrolled } \\
\text { seizures, mild speech comprehension } \\
\text { deficit }\end{array}$ & Rt insula \& TL/All & $73.0 / 6.2 / 91.5$ & $\begin{array}{l}\text { No new deficit, seizure free, speech } \\
\text { comprehension normalized }\end{array}$ \\
\hline 6 & $55, \mathrm{~F}$ & Rt/seizures & Rt insula, FL, \& TL/All & $82.0 / 1.5 / 98.2$ & No new deficit \\
\hline
\end{tabular}

All = astrocytoma Grade II; EOR = extent of resection (based on postoperative FLAIR sequence); FL = frontal lobe; OII = oligodendroglioma Grade II; TL = temporal lobe; $\mathrm{tV}=$ tumor volume. 



FIG. 3. Intraoperative visualization of LSAs during 6 consecutive awake resections of insular LGGs. A1-A6: Preoperative MRI of Cases 1-6. B1-B6: 3DUS power Doppler visualization of LSAs (arrows) in Cases 1-6.

a week, despite pharmacological treatment. MRI displayed a right-sided nonenhancing hyperintense temporoinsular glioma (Fig. 5A and B), and the LSAs were visualized by a contrast-enhanced 3D TOF sequence (Fig. 5C). Bilateral hemispheric dominance was revealed by functional MRI. The origin of the first LSA was located close to the middle cerebral artery (MCA) bifurcation (Fig. 6A-D). The preoperative speech and language investigation revealed a mild speech comprehension deficit.

A 2-stage resection was performed, as described above. During the second (awake) surgery, speech and language as well as motor functions were monitored. Before the removal of the insular tumor portion, the MCA and the first LSA were visualized using 3DUS power Doppler. We found the quality of imaging to be similar to the preoperative 3D TOF MRI sequence (Fig. 6E-H). The perforator was subsequently exposed in the expected location (Fig. 6I). Throughout the resection, the distance between the bottom of the resection cavity and the LSAs was repeatedly evaluated (Fig. 7) to avoid inadvertent exposure (and potential injury) of these perforators. Stimulation close to the posterior arm of the internal capsule elicited paresthesias of the left extremities. A small tumor remnant was intentionally left in place in this area, as well as a thin rim of infiltrated tissue over the LSAs (Fig. 5D-F). No motor or speech deficit was observed during the surgery.

After the procedure, the epileptic seizures completely resolved. A $92 \%$ resection was achieved. The patient returned to a normal social and professional life.

\section{Discussion}

The interval to a malignant transformation of insular LGGs is longer in patients who have undergone a greater extent of resection. ${ }^{15}$ Nevertheless, because permanent deficits have an adverse impact on the quality of life, which can well outweigh any prolongation of survival, ${ }^{16}$ the preservation of eloquent brain areas and important vessels is mandatory.

Resection of portions of an insular glioma that are adjacent to LSAs may result in injury of these vessels. The risk of LSA disruption is prominent, particularly when firm tumors are removed using surgical aspirators, which may damage the arterial wall. ${ }^{12}$ Moreover, suction apparatus can injure LSAs as well. ${ }^{12}$ Therefore, preoperative and especially intraoperative identification of LSA position is important.

Methods enabling preoperative visualization of LSAs and their anatomical relationship with the tumor were described; $;, 14$ these techniques are important, especially for planning the surgical strategy. However, the crucial step is intraoperative identification of LSAs. Several approaches to localizing these perforators and evaluation of the deepest limit of feasible resection have been reported.

First, anatomical landmarks are the basis for a surgeon's orientation. The plane defined by the bases of the periinsular sulci and the first perforating (most lateral) LSA helps to delineate the deepest part of the dissection., ${ }^{3,7}$ Unfortunately, apart from Yaşargil Type 3A insular glio- 

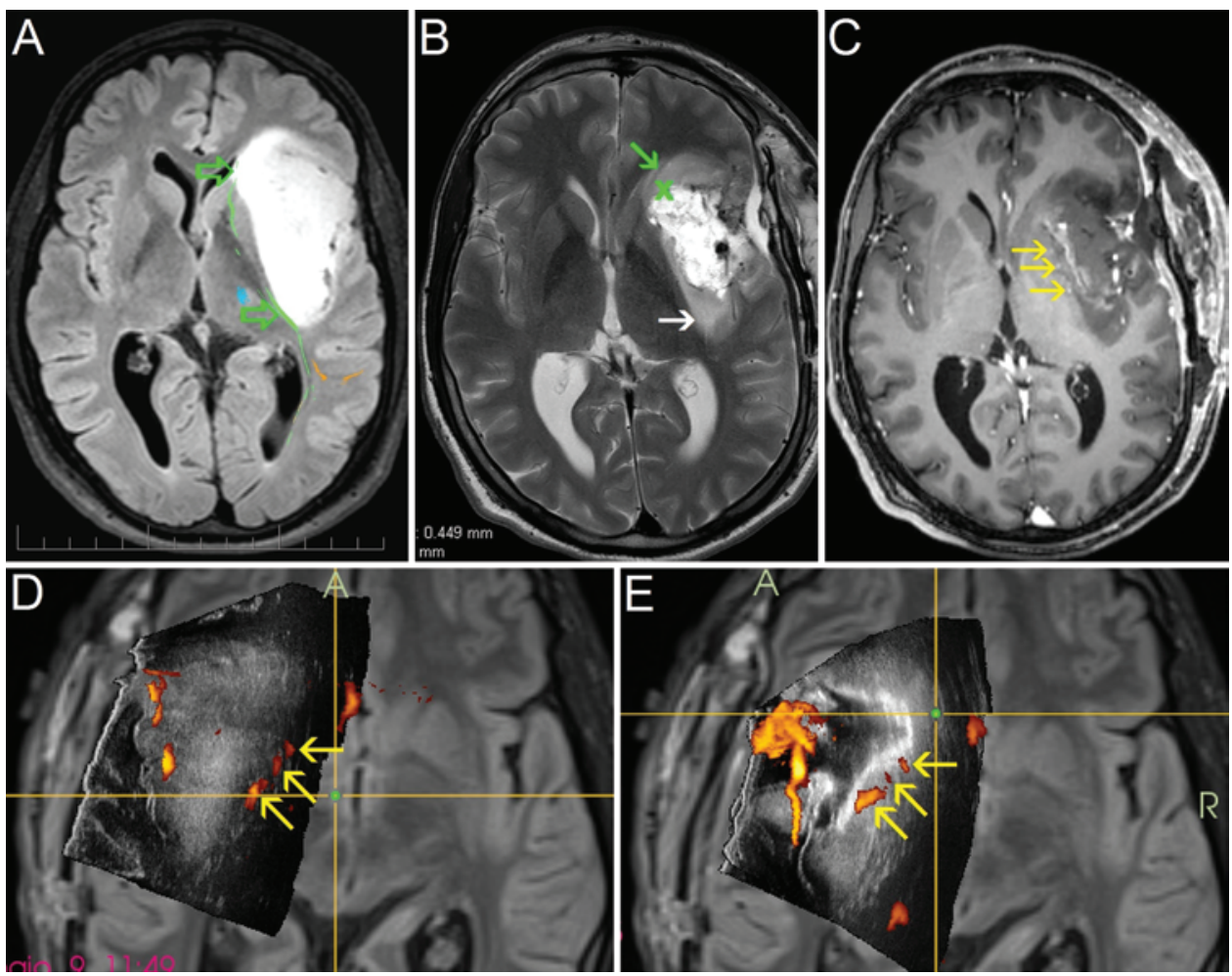

FIG. 4. Case 4. A: Preoperative axial FLAIR MRI sequence fused with tractography displaying the pyramidal tract (blue), arcuate fascicle (orange), and inferior frontooccipital fascicle (green), which is invaded by the tumor (arrows). B: Postoperative axial T2weighted MRI sequence showing partial resection. Green arrow = residual tumor involving the inferior frontooccipital fascicle; white arrow $=$ posteromedial part of the tumor (the dominant operculum hangs over this part); $\mathrm{X}=$ location where stimulation elicited semantic paraphasia. C: Postoperative axial contrast-enhanced T1-weighted MRI sequence showing LSAs (arrows). D: Initial 3DUS image fused with the preoperative MRI navigation. Arrows = LSAs depicted by power Doppler. E: Updated 3DUS image. Note the LSAs (arrows) just under the resection cavity.

mas, ${ }^{20,22}$ these anatomical landmarks are not sufficient for the surgeon to know with reliability the exact location of the perforating arteries on their whole trajectory. ${ }^{2}$ The nutmeg appearance of the striatum is another landmark that should be familiar to the surgeon; ${ }^{12,21}$ the lateral surface of the putamen is actually the place at which the surgeon has to stop the deep resection. ${ }^{18}$ However, LSAs are often encountered before the striatum. ${ }^{12}$ In addition, LGGs may involve the striatum, making its identification difficult; ${ }^{2}$ in the series published by Moshel et al., $34 \%$ of the insular gliomas extended medially to the displaced LSAs into the basal ganglia. ${ }^{9}$ The deep functional limit of resection is formed by eloquent white matter tracts (pyramidal tract, arcuate fasciculus, and inferior frontooccipital fasciculus), which can be identified by direct electrical subcortical stimulation. ${ }^{2}$

Another option for defining the deepest resection plane is utilization of neuronavigation systems that may be used for intraoperative medial tumor border identification.,12 Being familiar with the relationship of LSAs and the medial tumor border, the surgeon can indirectly evaluate the position of LSAs. In addition, some MRI sequences that can be used for neuronavigation, such as 3D TOF sequence, may directly visualize LSAs. Nevertheless, after some tumor debulking, neuronavigation becomes inaccurate and unreliable due to brain shift. ${ }^{10}$ An intraoperative update of navigational data using intraoperative MRI may be a plausible solution.

A method enabling direct localization of LSAs has been reported by Yaşargil et al.; the identification of LSAs is based on differentiation of the audio signal of intraoperative micro-Doppler recordings. ${ }^{21}$ In this regard, our technique uses a similar principle, but instead of localization based on an acoustic signal we use direct visualization of LSAs by power Doppler imaging. In addition to imaging of perforating arteries, 3DUS enables visualization of residual LGGs almost in real time; the fast 3DUS anatomical and power-Doppler data update makes this method also suitable for procedures with limited time such as awake resections. ${ }^{11,17}$ To the best of our knowledge, intraoperative visualization of LSAs by 3DUS power Doppler has not been previously described. Surprisingly, negative experiences were reported. ${ }^{2,5}$

Despite our promising results, 3 limitations of our work have to be considered. First, it is questionable how many LSAs in a given patient can be displayed by 3DUS power Doppler. In their anatomical study, Türe et al. reported the finding of 1-15 LSAs per hemisphere (average 7.75); while the diameters of the LSAs ranged between 0.1 and $1.5 \mathrm{~mm}$ (average $0.45 \mathrm{~mm}$ ), the majority (73\%) of LLAs measured less than $0.5 \mathrm{~mm}$ in diameter. ${ }^{19}$ However, 1.5-T MRI is not sufficient to display LSAs clearly enough, and even 



FIG. 5. Case 5. A and B: Preoperative axial T2-weighted MRI sequence showing a hyperintense temporoinsular tumor. C: Preoperative axial contrast-enhanced 3D TOF MRI sequence showing the position of the LSAs (arrows). D and E: Postoperative axial T2-weighted MRI sequence showing subtotal resection. A small rim of tumor tissue was intentionally left in place (arrows) to avoid injuring the LSAs. F: Postoperative axial contrast-enhanced 3D TOF MRI sequence showing the position of the LSAs (arrows) just under the resection cavity.

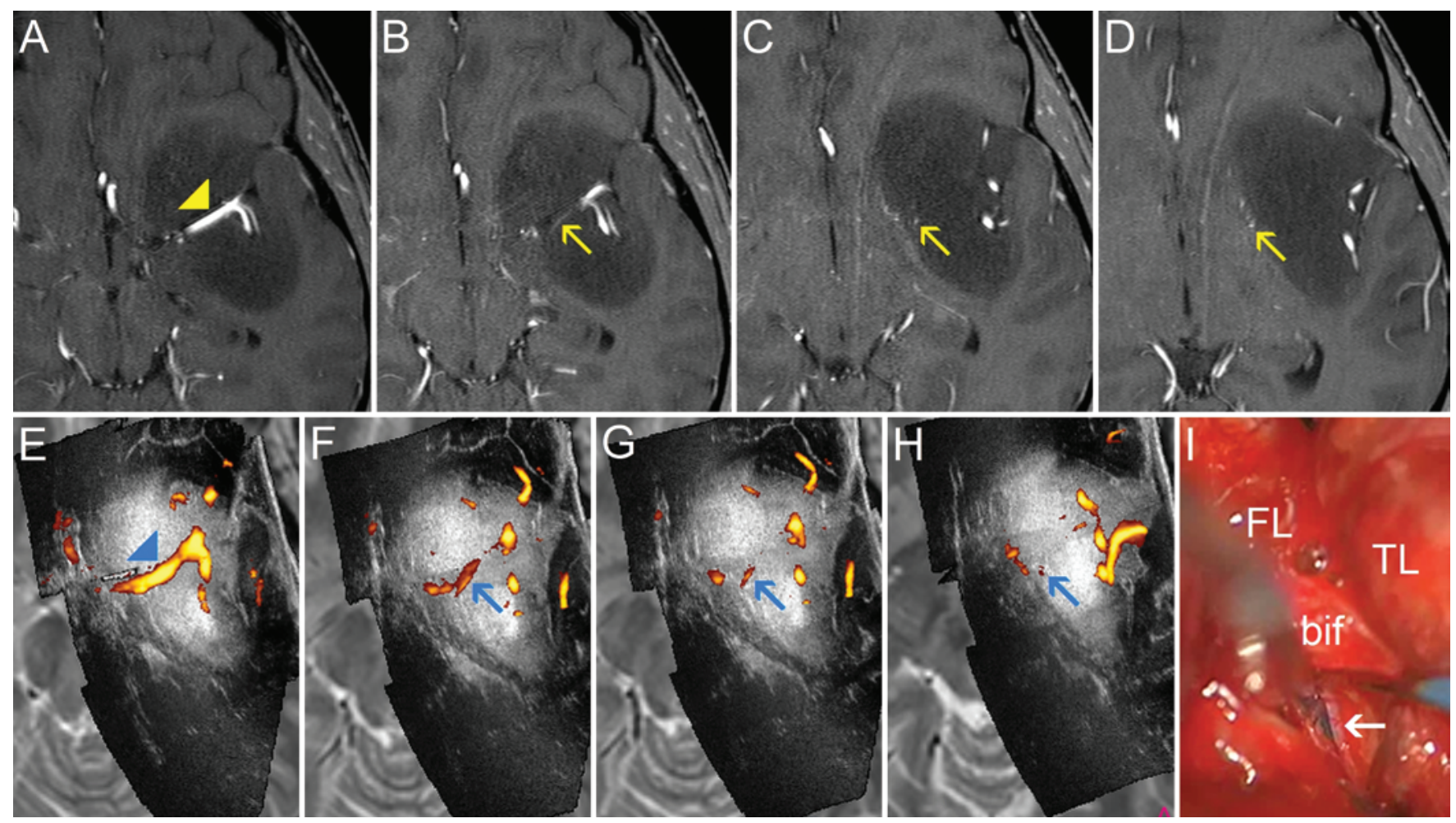

FIG. 6. Case 5. A: Preoperative axial contrast-enhanced 3D TOF MRI sequence showing the tumor-arterial relationship (the left-right orientation of the MR image was reversed for easier comparison with intraoperative 3DUS images). Arrowhead=

MCA. B-D: Trajectory of the first LSA (arrows). E: 3DUS image fused with the MRI navigation sequence before resection. Arrowhead = MCA displayed by power Doppler. F-H: Trajectory of the first LSA (arrows). I: Intraoperative photograph showing the first LSA (arrow). bif = MCA bifurcation; FL = frontal lobe; TL = temporal lobe. 



FIG. 7. Case 5. Intraoperative visualization of the LSAs during surgery. Intraoperative views before the resection (A), after the beginning of resection (B), and during the resection (C). Note that this allows the evaluation of the distance (purple line) between the bottom of the resection cavity and the LSAs.

3-T MRI scanners are unable to depict at least 1 LSA in every patient. ${ }^{1}$ Interestingly, 7-T MRI scanners using highresolution 3D TOF MR angiography are not able to depict all LSAs; perforators with diameters less than $250 \mu \mathrm{m}$ cannot be visualized. ${ }^{8}$ Implicitly, the number of LSAs that can be visualized by 3DUS power Doppler is uncertain. A prospective study of this topic is ongoing at our center. Second, even though power Doppler imaging is considered to be relatively angle independent,,$^{13}$ it is not completely angle independent; according to our experience it may be problematic to depict the whole course of LSAs. Again, this topic is being investigated in a prospective study. Future use of 3DUS with ultrasound contrast agents (which is an angle-independent method) could probably contribute to even better LSA visualization. Lastly, to quantify the reliability and effectiveness of the presented method, prospective studies are necessary to compare the extent of tumor resection and functional outcome of patients operated on with this technique versus other methods such as utilization of ultrasound without vascular imaging, MRIbased conventional neuronavigation alone, or handheld micro-Doppler.

In spite of these limitations, in all 6 operated cases the visualization of LSAs by 3DUS power Doppler was feasible and helped us prevent injury of these important perforators.

\section{Conclusions}

Visualization of LSAs by 3DUS power Doppler appears to be a useful tool during resection of insular LGGs. However, the reliability of this method has to be carefully evaluated in prospective studies.

\section{Acknowledgments}

This work was supported by a grant from the Scientific Grant Agency of the Ministry of Education of the Slovak Republic and the Slovak Academy of Sciences (VEGA; grant no. 1/0959/16).

\section{References}

1. Akashi T, Taoka T, Ochi T, Miyasaka T, Wada T, Sakamoto $\mathrm{M}$, et al: Branching pattern of lenticulostriate arteries observed by MR angiography at 3.0 T. Jpn J Radiol 30:331335, 2012

2. Duffau H: A personal consecutive series of surgically treated 51 cases of insular WHO Grade II glioma: advances and limitations. J Neurosurg 110:696-708, 2009

3. Hentschel SJ, Lang FF: Surgical resection of intrinsic insular tumors. Neurosurgery 57 (1 Suppl):176-183, 2005

4. Huncke K, Van de Wiele B, Fried I, Rubinstein EH: The asleep-awake-asleep anesthetic technique for intraoperative language mapping. Neurosurgery 42:1312-1317, 1998

5. Jakola AS, Berntsen EM, Christensen P, Gulati S, Unsgård G, Kvistad KA, et al: Surgically acquired deficits and diffusion weighted MRI changes after glioma resection-a matched case-control study with blinded neuroradiological assessment. PLoS One 9:e101805, 2014

6. Kumabe T, Nakasato N, Suzuki K, Sato K, Sonoda Y, Kawagishi J, et al: Two-staged resection of a left frontal astrocytoma involving the operculum and insula using intraoperative neurophysiological monitoring-case report. Neurol Med Chir (Tokyo) 38:503-507, 1998

7. Lang FF, Olansen NE, DeMonte F, Gokaslan ZL, Holland EC, Kalhorn C, et al: Surgical resection of intrinsic insular tumors: complication avoidance. J Neurosurg 95:638-650, 2001

8. Liem MK, van der Grond J, Versluis MJ, Haan J, Webb AG, Ferrari MD, et al: Lenticulostriate arterial lumina are normal in cerebral autosomal-dominant arteriopathy with subcortical infarcts and leukoencephalopathy: a high-field in vivo MRI study. Stroke 41:2812-2816, 2010

9. Moshel YA, Marcus JD, Parker EC, Kelly PJ: Resection of insular gliomas: the importance of lenticulostriate artery position. J Neurosurg 109:825-834, 2008

10. Nimsky C, Ganslandt O, Cerny S, Hastreiter P, Greiner G, Fahlbusch R: Quantification of, visualization of, and compensation for brain shift using intraoperative magnetic resonance imaging. Neurosurgery 47:1070-1080, 2000

11. Nossek E, Korn A, Shahar T, Kanner AA, Yaffe H, Marcovici $\mathrm{D}$, et al: Intraoperative mapping and monitoring of the corticospinal tracts with neurophysiological assessment and 3-dimensional ultrasonography-based navigation. Clinical article. J Neurosurg 114:738-746, 2011 
12. Rey-Dios R, Cohen-Gadol AA: Technical nuances for surgery of insular gliomas: lessons learned. Neurosurg Focus 34(2):E6, 2013

13. Rubin JM, Bude RO, Carson PL, Bree RL, Adler RS: Power Doppler US: a potentially useful alternative to mean frequency-based color Doppler US. Radiology 190:853-856, 1994

14. Saito R, Kumabe T, Inoue T, Takada S, Yamashita Y, Kanamori M, et al: Magnetic resonance imaging for preoperative identification of the lenticulostriate arteries in insular glioma surgery. Technical note. J Neurosurg 111:278-281, 2009

15. Sanai N, Polley MY, Berger MS: Insular glioma resection: assessment of patient morbidity, survival, and tumor progression. J Neurosurg 112:1-9, 2010

16. Simon M, Neuloh G, von Lehe M, Meyer B, Schramm J: Insular gliomas: the case for surgical management. J Neurosurg 110:685-695, 2009

17. Steňo A, Karlík M, Mendel P, Cík M, Steňo J: Navigated three-dimensional intraoperative ultrasound-guided awake resection of low-grade glioma partially infiltrating optic radiation. Acta Neurochir (Wien) 154:1255-1262, 2012

18. Türe U, González-López P: Glioma resection in eloquent areas: anatomical basis and resection with tractography studies, in Al-Mefty O (ed): Controversies in Neurosurgery II. Stuttgart: Thieme, 2014, pp 188-208

19. Türe U, Yaşargil MG, Al-Mefty O, Yaşargil DC: Arteries of the insula. J Neurosurg 92:676-687, 2000

20. Yaşargil DC: Microneurosurgery, Vol 4. Stuttgart: Thieme, 1994
21. Yaşargil MG, Krisht AF, Türe U, Al-Mefty O, Yaşargil D: Microsurgery of insular gliomas: Part IV: Surgical treatment and outcome. Contemporary Neurosurg 24:1-8, 2002

22. Yaşargil MG, von Ammon K, Cavazos E, Doczi T, Reeves JD, Roth P: Tumours of the limbic and paralimbic systems. Acta Neurochir (Wien) 118:40-52, 1992

\section{Disclosures}

The authors report no conflict of interest concerning the materials or methods used in this study or the findings specified in this paper.

\section{Author Contributions}

Conception and design: A Šteňo, J Šteňo. Acquisition of data: all authors. Analysis and interpretation of data: all authors. Drafting the article: A Šteňo. Critically revising the article: all authors. Reviewed submitted version of manuscript: all authors. Approved the final version of the manuscript on behalf of all authors: A Šteňo. Administrative/technical/material support: Jezberová, Hollý, Timárová. Study supervision: A Šteňo, J Šteňo.

\section{Correspondence}

Andrej Šteňo, Department of Neurosurgery, Comenius University, Faculty of Medicine, University Hospital Bratislava, Limbova 5,83305 Bratislava, Slovakia. email: andrej.steno@gmail.com. 